\title{
Management of Microvascular
}

\section{Complications in Secondary Diabetes Associated with Autoimmune Diseases - Case Report}

\author{
Enikő Nemes-Nagy¹, Robert Gabriel Tripon¹, Sándor Pál², Mariana Cornelia Tilinca³ \\ 1 Department of Biochemistry and Chemistry of Environmental Factors, University of Medicine and Pharmacy, Tîrgu Mureș, Romania \\ 2 University of Medicine and Pharmacy, Tîrgu Mureș, Romania \\ 3 Department of Cellular and Molecular Biology, University of Medicine and Pharmacy, Tîrgu Mureș, Romania
}

\section{CORRESPONDENCE \\ Robert Gabriel Tripon \\ Str. Gheorghe Marinescu nr. 38 \\ 540139 Tîrgu Mureș, Romania \\ Tel: +40749951988 \\ E-mail: robert.tripon@umftgm.ro}

\section{ARTICLE HISTORY}

Received: August 7, 2017

Accepted: August 21, 2017
Enikő Nemes-Nagy • Str. Gheorghe Marinescu nr. 38 540139 Tîrgu Mureș, Romania. Tel: +40 265215551

Sándor Pál • Str. Gheorghe Marinescu nr. 38, 540139 Tîrgu Mureș, Romania. Tel: +40 26521555

Mariana Cornelia Tilinca - Str. Gheorghe Marinescu nr. 38, 540139 Tîrgu Mureș, Romania. Tel: +40 265 215551

\begin{abstract}
The association of multiple autoimmune diseases may represent the main focus of physicians treating patients with such pathology presenting no comorbidities of different etiology. However, autoimmune diseases and side effects of drugs may lead to development of silent health-threatening diseases that should be identified promptly. We present the case of an elderly, obese, Caucasian female patient suffering of autoimmune thyroiditis, rheumatoid arthritis, and psoriasis, who developed arterial hypertension and insulin-treated secondary diabetes mellitus (due to long-term oral corticotherapy) with microvascular end-organ changes. Retinal imaging for capillary anomalies identified mild non-proliferative diabetic retinopathy with apparent diabetic macular edema and hypertensive retinopathy. Laboratory investigations looking for further vascular risk factors revealed zinc deficiency, elevated serum homocysteine levels, and constantly high C-reactive protein concentration. Attention should be payed to the proper investigation of patients with autoimmune diseases, targeting the early diagnosis of microvasculopathies due to autoimmune diseases or possible medication side effects, in order to prevent end-organ damage.
\end{abstract}

Keywords: autoimmune disease, diabetic retinopathy, laboratory investigations, microvascular complications, secondary diabetes mellitus

\section{INTRODUCTION}

Autoimmune diseases present a growing incidence worldwide. Data in the medical literature shows that autoimmune diseases often coexist in patients and their relatives. Statistics revealed that approximately half of the patients with autoimmune thyroid diseases report family members with thyroid disorders. One of the largest multi-centric studies that included over 3,000 Caucasian patients in the United Kingdom showed that patients with autoimmune thyroid diseases have a 
significantly increased relative risk for developing other autoimmune disorders, and this risk can be quantified. ${ }^{1}$

Psoriasis is a chronic skin disease associated with increased risk for type 2 diabetes mellitus, obesity, dyslipidemia, hypertension, and other cardiovascular diseases. Psoriatic arthritis occurs in $11-30 \%$ of the patients diagnosed with psoriasis. Rheumatoid arthritis (RA) is an autoimmune inflammatory disease of the joints; this condition is also associated with increased cardiovascular risk. ${ }^{2}$

Oral corticosteroids can be used for the management of several inflammatory and immunologic diseases, but their administration has serious negative consequences on the patient's state of health, especially on long-term usage and in high doses. The most important side effects of systemic corticotherapy include elevated serum glucose levels, adrenal suppression, dyslipidemia, weight gain, Cushing's syndrome, osteoporosis, cardiovascular disease, psychiatric diseases, and immunosuppression. ${ }^{3}$

Obesity induces a chronic inflammatory state and represents a major risk factor for the development of type 2 diabetes and ostheoarthritis. ${ }^{4}$

Tumor necrosis factor (TNF) inhibitors are modern therapeutic options for the treatment of chronic inflammation. Several recent studies focused on the effect of treatment on the development of comorbidities, such as impaired glucose tolerance and cardiovascular pathology in subjects with psoriasis and RA. Some studies revealed that TNF inhibitor therapy significantly decreased the risk for myocardial infarction and carbohydrate metabolic disorder in patients with psoriasis, psoriatic arthritis, and RA, but this effect is controversial according to other research data. $^{2}$

The aim of this case report is to present the development and assessment of microvascular complications in a patient with secondary diabetes mellitus due to corticotherapy treatment of multiple autoimmune diseases.

\section{CASE PRESENTATION}

We present the case of a 61-year-old obese Caucasian female patient admitted to the Procardia medical outpatient unit, where she was followed for five years. The patient was diagnosed after a stressful period in 2000 with RA, arterial hypertension, and tachycardia. Treatment with methotrexate for RA was initiated, but the therapy was later suspended because of skin rush and elevated serum transaminase activity revealing hepatic side effects. She received oral corticotherapy (prednisone), which led to secondary diabetes after three years. She is currently on insulin treatment ever since. In 2008, she was diagnosed with autoim- mune thyroiditis and in 2010 with psoriasis and psoriatic arthritis. According to her medical records, she has multiple allergies to different chemical substances.

\section{CLINICAL FINDINGS}

Our patient is hyperstenic, with grade 2 obesity (BMI $36.33 \mathrm{~kg} / \mathrm{m}^{2}$ ), and hypertensive, with a heart rate of 98 beats/min and a blood pressure of 140/95 mmHg. She currently presents pain at the level of several joints (radiocarpal, left metacarpophalangeal, and humeral joint, bilateral knees and ankles) and also psoriatic modifications of the skin, mostly at the level of the knees and elbows (Figure 1).

She developed neuropathy as a complication of type 2 diabetes, with the alteration of sensory perception at the level of the feet, and retinopathy due to hypertensive and diabetic microvascular complications.

\section{LABORATORY INVESTIGATIONS}

According to the latest laboratory results, our patient has a good carbohydrate metabolic balance, based on the glycated hemoglobin (HbAlc) value $(6.8 \%)$ obtained by reflectometry using a Nycocard Reader equipment, and the intensity of oxidative stress was in normal range, based on serum malondialdehyde (MDA) measurement using the thiobarbituric acid reactive substances(TBARS) method on high pressure liquid chromatography (HPLC): $7.13 \mathrm{ng} / \mathrm{mL}$ (normal under $8.2 \mathrm{ng} / \mathrm{mL}$ ). Signs of chronic inflammation can be observed, based on her constantly high C-reactive protein values $(2.21-1,400 \mathrm{mg} / \mathrm{dL}$, normal below $0.33 \mathrm{mg} / \mathrm{dL}$ ) and sometimes elevated blood sedimentation rate (BSR), up to $33 \mathrm{~mm} / \mathrm{h}$. Her transaminase values (GOT/GPT) were episodically slightly elevated, up to 64/115 U/L (normal under 37/40 U/L), and also gammaGT and alkaline phosphatase were sometimes discretely high (64 IU/L and $294 \mathrm{IU} / \mathrm{L}$, normal up to $32 \mathrm{IU} / \mathrm{L}$ and $279 \mathrm{IU} / \mathrm{L}$, respectively).

The patient's serum cholesterol value was slightly increased $(228 \mathrm{mg} / \mathrm{dL})$, serum creatinine was repeatedly in the normal range (1-1.05 $\mathrm{mg} / \mathrm{dL})$. Her kidney function was also investigated based on cystatin $\mathrm{C}$ measurement, an early marker of nephropathy, which was also in the normal range (1.19 mg/L, normal range $0.63-1.44 \mathrm{mg} / \mathrm{L})$. All these biochemical analyses were performed on a Konelab 20Xti analyzer.

She presented increased values of serum homocysteine $(20.6 \mu \mathrm{mol} / \mathrm{L}$, normal under $10 \mu \mathrm{mol} / \mathrm{L})$, and zinc deficiency $(6.99 \mu \mathrm{mol} / \mathrm{L}$, normal range $10.4-16.4 \mu \mathrm{mol} / \mathrm{L})$. She had an increased serum chromium concentration of 


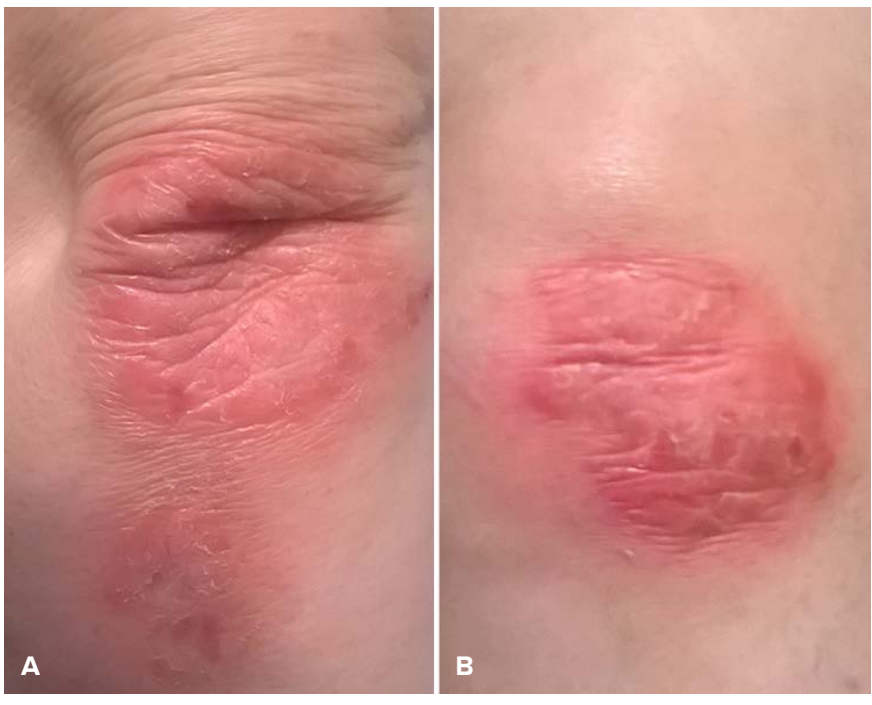

FIGURE 1. Psoriatic skin manifestations of the right elbow $(\mathbf{A})$ and left knee (B)

$2.72 \mu \mathrm{g} / \mathrm{L}$ (normal range $0.31-2.44 \mu \mathrm{g} / \mathrm{L}$ ) and an elevated serum vitamin $\mathrm{B} 12$ concentration $(1,200 \mathrm{pg} / \mathrm{mL}$, normal range 191-663 pg/mL). Immulite One equipment was used for homocysteine and vitamin B12 measurement by chemiluminescence methodology.

Under levothyroxine sodium treatment, her serum thyroid stimulating hormone (TSH) level is currently normal (3.48 $\mu \mathrm{IU} / \mathrm{mL}$, reference range $0.39-6.13 \mu \mathrm{IU} / \mathrm{mL}$ ) and her free thyroxine (FT4) is close to the lower limit, but still normal $(0.87 \mathrm{ng} / \mathrm{dL}$, reference range $0.80-2.00 \mathrm{ng} /$ dL). Her anti thyroid peroxidase antibody (ATPO) level showed repeatedly very high values, up to $485.93 \mathrm{IU} / \mathrm{L}$ (normal under $35 \mathrm{IU} / \mathrm{L}$ ).

\section{RETINAL IMAGING}

The patient was referred to the Ophthalmology Department of the Mureș County Clinical Hospital for complaints including eye redness and scratchy sensation in both eyes. A complete eye examination was performed. Carl Zeiss Visucam 500 fundus camera was used for retinal photography in the context of diabetic retinopathy screening and follow-up.

The patient's uncorrected visual acuity measured with the Early Treatment Diabetic Retinopathy Study (ETDRS) chart was 0.8 in both eyes (decimal scale), correctable to 1.0 best corrected visual acuity. No related history or clinical evidence for uveitis was identified. Schirmer's test revealed $5 \mathrm{~mm}$ (right eye) and $7 \mathrm{~mm}$ (left eye) of tear production on the filter paper in 5 minutes, identifying dry eyes responsible for the scratchy sensation described by the patient. Dilated ophthalmoscopy and retinal imaging revealed

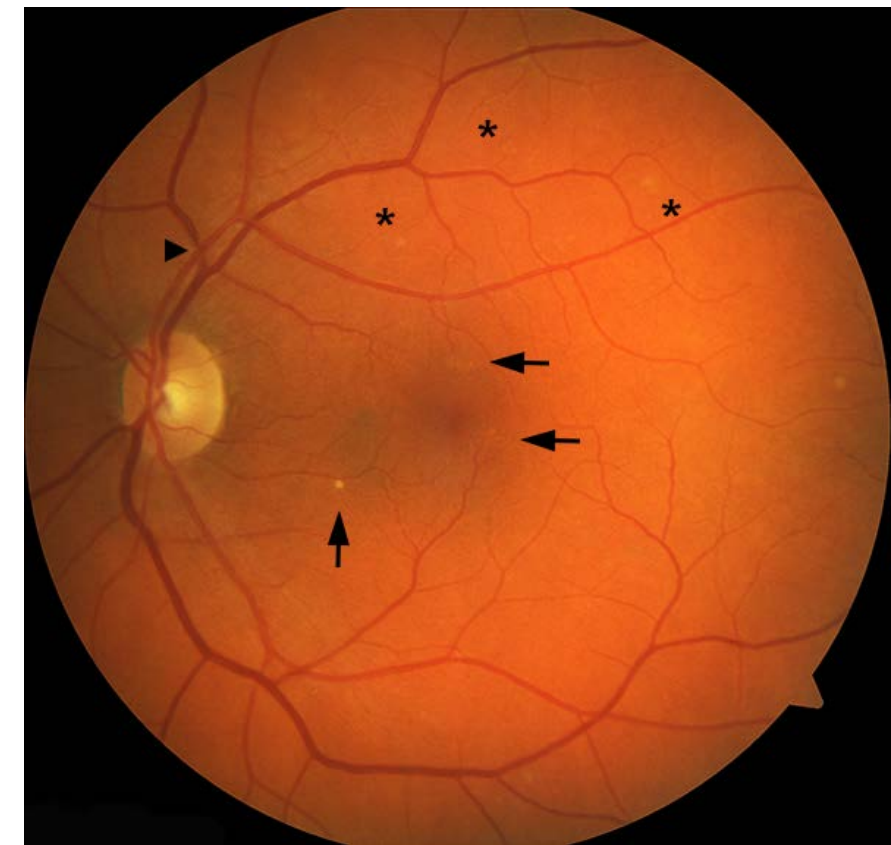

FIGURE 2. Mild non-proliferative diabetic retinopathy with maculopathy and grade II hypertensive retinopathy: leaking capillaries contribute to the formation of exudates in the perifovea and parafovea (arrowheads) and across the mid-peripheral retina (asterisk), as seen on the 45-degree angle fundus photography of the left eye. Vascular nipping due to chronic arterial hypertension is also present at arterio-venous crossing sites (triangle).

vascular caliber changes, nipping, micro-aneurysms, focal leakage of capillaries, and hard exudates in the macular region and peripheral retina in both eyes (Figure 2). Considering the ETDRS classification, the patient was diagnosed with mild non-proliferative diabetic retinopathy with diabetic macular edema (DMO) apparently present. Ocular coherence tomography for the macular regions of both eyes was suggested for an accurate evaluation of DMO, but the patient preferred to temporize any further investigation. Grade II hypertensive retinopathy was also identified (Keith-Wagner-Barker classification). In this context, the patient was explained the importance of targeting normal values of blood glucose level and blood pressure and the necessity of a follow-up fundus examination in 6 months, and natural tears were prescribed for daily instillation.

\section{THERAPEUTIC CHALLENGES}

After a few years of methotrexate given for her rheumatoid arthritis, which was not tolerated because of hepatotoxicity, our patient received oral prednisone treatment, then etanercept between 2008-2012, a TNF receptor inhibitor recommended in RA and also in psoriasis, which also had several side effects. Etanercept increases the risk for cer- 
tain types of cancer and weakens the immune system. Our patient's condition was notably improved, so the treatment was stopped to avoid side effects. Her current treatment, from April 2016, is with golimumab $50 \mathrm{mg} / \mathrm{month}$ (another TNF inhibitor with less side effects) and leflunomide (tablets of $20 \mathrm{mg}, 1 /$ day), an immunosuppressive antirheumatic drug. In case of acute pain, local non-steroid anti-inflammatory creams and diclofenac sodium suppository $(100 \mathrm{mg}, 2 \times 1 /$ day $)$ or aceclofenac tablets of $100 \mathrm{mg}$ $(2 \times 1$ tablets/day) were prescribed together with gastric protector (omeprazole $2 \times 20 \mathrm{mg} /$ day).

The management of patients with multiple pathologies is very difficult. Physicians need to apply special, individual therapy schemes, taking into consideration the adverse effects of the medication. Our patient could not receive oral antidiabetic drugs because of their potential side effects, so she was given insulin injections to control her type 2 diabetes mellitus. Her antidiabetic treatment consists of a basal bolus regimen with detemir insulin $0-0-58$ units/day and glulisine insulin 0-8-0 units/day for her secondary diabetes diagnosed in 2008, which was not responsive to 2 months of diet and phytotherapeutic products.

The patient's cardiovascular problems (including grade II hypertension with very high risk, tachycardia, and varicose veins) are treated with oral therapy using angiotensin-converting enzyme-inhibitors (enalapril $2 \times 20 \mathrm{mg} /$ day), diuretics (indapamid $1.5 \mathrm{mg} /$ day, spironolactone $100 \mathrm{mg}$, and furosemide $20 \mathrm{mg}$ every two days), antiplatelet (acetylsalicylic acid $75 \mathrm{mg} /$ day), non-dihydropyridine calcium channel blocker (diltiazem $2 \times 90 \mathrm{mg}$ /day), vasoprotective medication (diosminum $2 \times 500 \mathrm{mg} /$ day). The hypercholesterolemia was treated with rosuvastatin $10 \mathrm{mg}$ once a day. She also received 50 micrograms of levothyroxine sodium each day for her thyroid disorder, as well as benfotiamine and pyridoxine hydrochloride twice a day for her diabetic neuropathy.

The patient received nutritional counselling for obesity, diabetes, and arterial hypertension.

For the protection of liver cells, in order to counteract the hepatotoxicity of her current treatment, she received essential phospholipids and a dietary supplement with a complex hepatoprotective formula twice a day.

The patient agreed to the publication of her data and the institution where the patient had been admitted, approved the publication of the case.

\section{DISCUSSION}

Immune-related dry eye syndrome (IRDE) and ocular surface inflammation have been previously characterized and described in the context of rheumatoid arthritis, highlighting the necessity of targeted diagnostic investigations. Moreover, the progression of the disease was closely related to the ocular surface signs and symptoms. ${ }^{5}$ Our patient's positive Schirmer's test and subjective complaints helped to identify dry eye syndrome in accordance with the International Dry Eye Workshop classification, and proper treatment was initiated.

Although uveitis or vasculitis affecting the retinal vasculature are common in patients suffering of autoimmune diseases such as psoriasis or rheumatoid arthritis, our patient was unaffected. ${ }^{6}$

Vasculature integrity and pathology may be easily visualized and followed on retinal microvasculature during fundus examination. Yen et al. showed in a populationbased cohort study the association between psoriasis and an increased risk of developing retinal vein occlusion, and autoimmune thyroiditis was identified as a risk factor for stroke. ${ }^{7,8}$ Psoriasis and psoriatic arthritis have also been shown to represent an increased risk for developing diabetes, hypertension, obesity, and hyperlipidemia. ${ }^{9}$ Subsequently, screening for microvascular changes may be of high importance, preventing irreversible tissue damage in retinopathy, neuropathy, or nephropathy. The coexistence of diabetes mellitus, arterial hypertension, obesity, and dyslipidemia may worsen the vascular end-organ damage. ${ }^{10}$

Laboratory investigations identified multiple vascular risk factors such as serum zinc deficiency, elevated serum homocysteine levels, and constantly high C-reactive protein concentration. Hyper-homocysteinemia was associated with the development of thrombotic and atherosclerotic diseases and vascular leakage. ${ }^{11}$ Zinc was suggested to act on vascular permeability and is involved in the coagulation cascade, being also responsible for thrombotic events. ${ }^{12} \mathrm{C}$-reactive protein, homocysteine, and other inflammatory factors regulate endothelial cell response and consequently adhesion molecules, contributing to vascular leakage. These circulating inflammatory factors are also involved in the atherosclerotic pathology underlying inflammatory rheumatic diseases. ${ }^{10}$

We observed some particular features in case of the presented patient, probably in relationship with her diet (rich in nuts, peanuts, herbal teas) and dietary supplements: increased serum chromium and vitamin B12 concentration. Flavonoids and p-methoxybenzoic acid, contained in the composition of the herbal product Caper bush (Capparis L.) taken by the patient, attenuate oxidative stress and prevent the elevation of malondialdehyde. Other chemicals present in its composition include rutin, quercetin, and 
also steroids together with other antioxidant liposoluble compounds (tocopherols, carotenoids). ${ }^{13}$

Predisposition for autoimmune disorders is running in the family. Our patient's sister is currently treated with severe psoriasis, and her daughter had repeatedly high serum ATPO levels (between 300-400 IU/L), so far with normal TSH and FT4 concentrations.

\section{CONCLUSIONS}

Autoimmune diseases can be diagnosed in an early phase based on a wide range of laboratory tests recommended to be performed in the patient and family members. Early diagnosis increases the chance of proper treatment implemented in time and thus, the prevention of complications. However, microvascular changes may develop unnoticed, leading to irreversible tissue damage. Attention should be payed to the proper investigation of patients with autoimmune diseases, targeting the early diagnosis of microvasculopathies due to autoimmune diseases or possible medication side effects, in order to prevent end-organ damage.

\section{ACKNOWLEDGEMENT}

We are grateful to nurse with laboratory master degree Nyárádi Annamária for helping our research team in the assessment of detailed information regarding the patient's and her family's medical history.

\section{CONFLICT OF INTEREST}

Nothing to declare.

\section{REFERENCES}

1. Boelaert K, Newby PR, Simmonds MJ, et al. Prevalence and relative risk of other autoimmune diseases in subjects with autoimmune thyroid disease. Am J Med. 2010;123:183.e1-183.e9.

2. Wu JJ, Rowan CG, Bebchuk JD, Anthony MS. No Association Between TNF Inhibitor and Methotrexate Therapy Versus Methotrexate in Changes in Hemoglobin A1C and Fasting Glucose Among Psoriasis, Psoriatic Arthritis, and Rheumatoid Arthritis Patients. J Drugs Dermatol. 2015;14:159-166.

3. Liu D, Ahmet A, Ward L, et al. A practical quide to the monitoring and management of the complications of systemic corticosteroid therapy. Allergy Asthma Clin Immunol. 2013:9:30.

4. Aspden RM. Obesity punches above its weight in osteoarthritis. Nat Rev Rheumatol. 2011;7:65-68.

5. Wang $\mathrm{H}$, Wang PB, Chen $\mathrm{T}$, et al. Analysis of clinical characteristics of immune-related dry eye. J Ophthalmol. 2017;2017:8532397.

6. Abbouda A, Abicca I, Fabiani C, et al. Psoriasis and psoriatic arthritisrelated uveitis: different ophthalmological manifestations and ocular inflammation features. Semin Ophthalmol. 2016;15:1-6

7. Yen YC, Weng SF, Lai FJ, Lin YS. Risk of retinal vein occlusion in patients with psoriasis: A population-based cohort study. Retina. 2015;35:17861794.

8. Karch A, Thomas SL. Autoimmune thyroiditis as risk factor for stroke Neurology. 2014;82:1643-1652.

9. Mallbris L, Ritchlin CT, Stahle M. Metabolic disorders in patients with psoriasis and psoriatic arthritis. Curr Rheumatol Rep. 2006;8:355-363.

10. Szekanecz Z, Koch AE. Vascular involvement in rheumatic diseases: 'vascular rheumathology'. Arthriris Res Ther. 2008;10:224

11. Lentz SR. Homocysteine and vascular dysfunction. Life Sci. 1997;61:12051215.

12. Vu TT, Fredenburgh JC, Weitz JI. Zinc: an important cofactor in haemostasis and thrombosis. Thromb Haemost. 2013;109:421-430.

13. Tlili N, Elfalleh W, Saadaoui E, Khaldi A, Triki S, Nasri N. The caper (Capparis L.): Ethnopharmacology, phytochemical and pharmacological properties. Fitoterapia. 2011;82:93-101. 